Georgian Mathematical Journal

Volume 14 (2007), Number 3, 457-469

\title{
ON DOUBLY PERIODIC SOLUTIONS OF NONLINEAR HYPERBOLIC EQUATIONS OF HIGHER ORDER
}

\author{
TARIEL KIGURADZE
}

\begin{abstract}
Unimprovable conditions of the existence and uniqueness of doubly periodic solutions are established for nonlinear hyperbolic equations of higher order with two independent variables.
\end{abstract}

2000 Mathematics Subject Classification: 35L35, 35B10.

Key words and phrases: Nonlinear, higher order, hyperbolic equation, doubly periodic.

\section{Statement of the Problem and the Main Results}

Consider the nonlinear hyperbolic equation

$$
\begin{gathered}
u^{(2 m, 2 n)}=\sum_{k=0}^{n-1}\left(a_{m k} u^{(2 m, 2 k)}+b_{m k} u^{(2 m, 2 k+1)}\right)+\sum_{i=0}^{m-1}\left(a_{i n} u^{(2 i, 2 n)}+c_{i n} u^{(2 i+1,2 n)}\right) \\
+\sum_{i=0}^{m-1} \sum_{k=0}^{n-1}\left(a_{i k} u^{(2 i, 2 k)}+b_{i k} u^{(2 i, 2 k+1)}+c_{i k} u^{(2 i+1,2 k)}\right)+f(x, y, u)
\end{gathered}
$$

where $a_{i k}, b_{i k}, c_{i k}$ are real constants, $f: \mathbb{R}^{3} \rightarrow \mathbb{R}$ is a continuous function, and for any $i$ and $k$

$$
u^{(i, k)}(x, y)=\frac{\partial^{i+k} u(x, y)}{\partial x^{i} \partial y^{k}}
$$

A function $u: \mathbb{R}^{2} \rightarrow \mathbb{R}$ is called a solution of equation (1.1) if it is continuous together with all its derivatives $u^{(i, j)}(i=0,1, \ldots, 2 m ; k=0,1, \ldots, 2 n)$ and satisfies equation (1.1) everywhere in $\mathbb{R}^{2}$.

Let $\omega_{1}$ and $\omega_{2}$ be positive numbers. A solution $u$ of equation (1.1) is called $\left(\omega_{1}, \omega_{2}\right)$-periodic if

$$
u\left(x+\omega_{1}, y\right)=u(x, y), \quad u\left(x, y+\omega_{2}\right)=u(x, y) \quad \text { for } \quad(x, y) \in \mathbb{R}^{2} .
$$

Problems on the existence of $\left(\omega_{1}, \omega_{2}\right)$-periodic solutions to hyperbolic equations of second and fourth orders were studied in [1-4,8-14], and for higher order equations in [6]. In the present paper new, unimprovable in a sense, conditions of existence and uniqueness of $\left(\omega_{1}, \omega_{2}\right)$-solutions are established.

Naturally, we assume that $f$ is $\left(\omega_{1}, \omega_{2}\right)$-periodic with respect to the first two variables, i.e., the following equalities hold in $\mathbb{R}^{3}$

$$
f\left(x+\omega_{1}, y, z\right)=f(x, y, z), \quad f\left(x, y+\omega_{2}, z\right)=f(x, y, z) .
$$

2000 Mathematics Subject Classification. 
Theorem 1.1. Let

$$
\begin{aligned}
(-1)^{m+n+i+k} a_{i k} \geq 0 \quad & (i=0, \ldots, m ; k=0, \ldots, n ; \\
& i+k<m+n), \quad a_{0 n} \neq 0, \quad a_{m 0} \neq 0,
\end{aligned}
$$

and let there exist positive constants $a, b$ and $c$ such that

$$
\begin{gathered}
\sum_{k=0}^{n-1}\left(\frac{\omega_{2}}{2 \pi}\right)^{2 n-2 k}\left|a_{m k}\right|+\sum_{i=0}^{m-1}\left(\frac{\omega_{1}}{2 \pi}\right)^{2 m-2 i}\left|a_{i n}\right| \\
+\sum_{i=0}^{m-1} \sum_{k=0}^{n-1}\left(\frac{\omega_{1}}{2 \pi}\right)^{2 m-2 i}\left(\frac{\omega_{2}}{2 \pi}\right)^{2 n-2 k}\left|a_{i k}\right|+\left(\frac{\omega_{1}}{2 \pi}\right)^{m}\left(\frac{\omega_{2}}{2 \pi}\right)^{n} b^{\frac{1}{2}}<1, \\
a|z|-c \leq(-1)^{m+n} f(x, y, z) \leq b|z|+c \text { for }(x, y, z) \in \mathbb{R}^{3} .
\end{gathered}
$$

Then equation (1.1) has at least one $\left(\omega_{1}, \omega_{2}\right)$-periodic solution.

Theorem 1.2. Let inequalities (1.2) and (1.3) hold, and

$$
a\left|z_{1}-z_{2}\right| \leq(-1)^{m+n}\left(f\left(x, y, z_{1}\right)-f\left(x, y, z_{2}\right)\right) \leq b\left|z_{1}-z_{2}\right|,
$$

where $a$ and $b$ are positive constants. Then equation (1.1) has one and only one $\left(\omega_{1}, \omega_{2}\right)$-periodic solution.

For higher order ordinary differential equations results similar to Theorems 1.1 and 1.2 were obtained by I. Kiguradze and T. Kusano in [7].

Example 1.1. Let $\varepsilon \in(0,1)$ be an arbitrarily small number and

$$
\delta=\frac{1-\sqrt{1-\varepsilon}}{2} .
$$

Consider the differential equation

$$
\begin{gathered}
u^{(2 m, 2 n)}=\sum_{k=0}^{n-1} a_{m k} u^{(2 m, 2 k)}+\sum_{i=0}^{m-1} a_{i n} u^{(2 i, 2 n)} \\
+\sum_{i=1}^{m-1} \sum_{k=1}^{n-1} a_{i k} u^{(2 i, 2 k)}+(-1)^{m+n} b u+\sin \frac{2 \pi x}{\omega_{1}} \sin \frac{2 \pi y}{\omega_{2}},
\end{gathered}
$$

where $a_{i k}(i=0, \ldots, m ; k=0, \ldots, n ; 2 \leq i+k<m+n)$ are constants satisfying inequalities (1.2), and

$$
\begin{gathered}
\sum_{k=0}^{n-1}\left(\frac{\omega_{2}}{2 \pi}\right)^{2 n-2 k}\left|a_{m k}\right|+\sum_{i=0}^{m-1}\left(\frac{\omega_{1}}{2 \pi}\right)^{2 m-2 i}\left|a_{i n}\right| \\
+\sum_{i=0}^{m-1} \sum_{k=0}^{n-1}\left(\frac{\omega_{1}}{2 \pi}\right)^{2 m-2 i}\left(\frac{\omega_{2}}{2 \pi}\right)^{2 n-2 k}\left|a_{i k}\right|=1-\delta^{2}, \quad b=\delta^{2}\left(\frac{2 \pi}{\omega_{1}}\right)^{2 m}\left(\frac{2 \pi}{\omega_{2}}\right)^{2 n} .
\end{gathered}
$$


It is clear that equation (1.6) satisfies all of the conditions of Theorems 1.1 and 1.2 except condition (1.3). Instead of (1.3) it satisfies the condition

$$
\begin{gathered}
\sum_{k=0}^{n-1}\left(\frac{\omega_{2}}{2 \pi}\right)^{2 n-2 k}\left|a_{m k}\right|+\sum_{i=0}^{m-1}\left(\frac{\omega_{1}}{2 \pi}\right)^{2 m-2 i}\left|a_{i n}\right| \\
+\sum_{i=0}^{m-1} \sum_{k=0}^{n-1}\left(\frac{\omega_{1}}{2 \pi}\right)^{2 m-2 i}\left(\frac{\omega_{2}}{2 \pi}\right)^{2 n-2 k}\left|a_{i k}\right|+\left(\frac{\omega_{1}}{2 \pi}\right)^{m}\left(\frac{\omega_{2}}{2 \pi}\right)^{n} b^{\frac{1}{2}}<1+\varepsilon .
\end{gathered}
$$

Let us show that equation (1.1) has no $\left(\omega_{1}, \omega_{2}\right)$-periodic solution. Assume the contrary that such a solution exists. Then

$$
\begin{gathered}
\int_{0}^{\omega_{1}} \int_{0}^{\omega_{2}} \sin \frac{2 \pi x}{\omega_{1}} \sin \frac{2 \pi y}{\omega_{2}} u^{(2 i, 2 k)}(x, y) d x d y \\
=(-1)^{i+k}\left(\frac{2 \pi}{\omega_{1}}\right)^{2 i}\left(\frac{2 \pi}{\omega_{2}}\right)^{2 k} \int_{0}^{\omega_{1}} \int_{0}^{\omega_{2}} \sin \frac{2 \pi x}{\omega_{1}} \sin \frac{2 \pi y}{\omega_{2}} u(x, y) d x d y .
\end{gathered}
$$

Multiplying both sides of equation (1.6) by $\sin \frac{2 \pi x}{\omega_{1}} \sin \frac{2 \pi y}{\omega_{2}}$, integrating over $\left[0, \omega_{1}\right] \times\left[0, \omega_{2}\right]$ and taking into account (1.7) and (1.9), we get the contradiction

$$
\int_{0}^{\omega_{1}} \int_{0}^{\omega_{2}} \sin ^{2} \frac{2 \pi x}{\omega_{1}} \sin ^{2} \frac{2 \pi y}{\omega_{2}} d x d y=0 .
$$

The constructed example shows that in Theorems 1.1 and 1.2 inequality (1.3) cannot be replaced by inequality (1.9) whatever small $\varepsilon>0$ may be.

Theorem 1.3. Let

$$
\begin{aligned}
(-1)^{m+n+i+k} a_{i k} \leq 0 \quad & (i=0, \ldots, m ; k=0, \ldots, n \\
& i+k<m+n), \quad a_{0 n} \neq 0, \quad a_{m 0} \neq 0,
\end{aligned}
$$

and let there exist a positive constant $c$ such that

$$
(-1)^{m+n} f(x, y, z) z<0 \text { for }(x, y) \in \mathbb{R}^{2},|z|>c .
$$

Then equation (1.1) has at least one $\left(\omega_{1}, \omega_{2}\right)$-periodic solution.

Example 1.2. Consider the differential equation

$$
\begin{aligned}
u^{(2 m, 2 n)} & =\sum_{k=0}^{n-1} a_{m k} u^{(2 m, 2 k)}+\sum_{i=0}^{m-1} a_{i n} u^{(2 i, 2 n)} \\
+ & \sum_{i=1}^{m-1} \sum_{k=1}^{n-1} a_{i k} u^{(2 i, 2 k)}+\frac{1}{1+u^{2}},
\end{aligned}
$$

where $a_{i k}(i=0, \ldots, m ; k=0, \ldots, n ; 2 \leq i+k<m+n)$ are the constants satisfying inequalities (1.10). Equation (1.12) satisfies all the conditions of 
Theorem 1.3 except condition (1.11). Instead of (1.11) it satisfies the condition

$$
\limsup _{|z| \rightarrow+\infty}(-1)^{m+n} f(x, y, z) \leq 0 \quad \text { for } \quad(x, y) \in \mathbb{R}^{2} .
$$

On the other hand, it is clear that equation (1.12) has no $\left(\omega_{1}, \omega_{2}\right)$-periodic solution for any positive $\omega_{1}$ and $\omega_{2}$. This example shows that in Theorem 1.3 condition (1.11) is optimal and cannot be replaced by condition (1.13).

Theorem 1.4. Let all of the conditions of Theorem 1.3 hold and

$$
(-1)^{m+n}\left(f\left(x, y, z_{1}\right)-f\left(x, y, z_{2}\right)\right)\left(z_{1}-z_{2}\right)<0 \text { for }(x, y) \in \mathbb{R}^{2}, z_{1} \neq z_{2} \text {. }
$$

Then equation (1.1) has one and only one $\left(\omega_{1}, \omega_{2}\right)$-periodic solution.

In contrast to Theorems 1.1 and 1.2, Theorems 1.3 and 1.4 do not restrict the growth order of the function $f$ with respect to the third argument. For example, the function

$$
f(x, y, z)=(-1)^{m+n+1} p_{0}(x, y) \exp \left(p_{1}(x, y) z^{2}\right) z^{2 l-1}+q(x, y),
$$

where $p_{0}: \mathbb{R}^{2} \rightarrow(0,+\infty), p_{1}: \mathbb{R}^{2} \rightarrow[0,+\infty)$ and $q: \mathbb{R}^{2} \rightarrow \mathbb{R}$ are arbitrary continuous $\left(\omega_{1}, \omega_{2}\right)$-periodic functions and $l$ is an arbitrary natural number, satisfies conditions (1.11) and (1.14).

Example 1.3. Let inequalities (1.10) hold and

$$
f(x, y, z)=(-1)^{m+n+1} p_{0}(x, y) f_{0}(z)
$$

where

$$
f_{0}(z)=\left\{\begin{array}{l}
0 \text { for }|z| \leq \delta \\
z-\delta \operatorname{sgn} z \text { for }|z|>\delta
\end{array}\right.
$$

$\delta$ is a positive constant, and $p_{0}: \mathbb{R}^{2} \rightarrow(0,+\infty)$ is a continuous $\left(\omega_{1}, \omega_{2}\right)$-periodic function. Then it is clear that $f$ satisfies condition (1.11), where $c>\delta$. However, instead of (1.14) $f$ satisfies the condition

$$
(-1)^{m+n}\left(f\left(x, y, z_{1}\right)-f\left(x, y, z_{2}\right)\right)\left(z_{1}-z_{2}\right) \leq 0 \quad \text { for } \quad(x, y) \in \mathbb{R}^{2} .
$$

On the other hand, it is clear that for any $\gamma \in[-\delta, \delta]$ the constant function $u(x, y)=\gamma$ is a $\left(\omega_{1}, \omega_{2}\right)$-periodic solution of equation (1.1). Thus we have shown that in Theorem 1.4 condition (1.14) cannot be replaced by (1.15).

The equation

$$
\begin{gathered}
u^{(2 m, 2 n)}=\sum_{k=0}^{n-1}\left(a_{m k} u^{(2 m, 2 k)}+b_{m k} u^{(2 m, 2 k+1)}\right)+\sum_{i=0}^{m-1}\left(a_{i n} u^{(2 i, 2 n)}+c_{i n} u^{(2 i+1,2 n)}\right) \\
+\sum_{i=0}^{m-1} \sum_{k=0}^{n-1}\left(a_{i k} u^{(2 i, 2 k)}+b_{i k} u^{(2 i, 2 k+1)}+c_{i k} u^{(2 i+1,2 k)}\right)+p(x, y) u+q(x, y)
\end{gathered}
$$

is a particular case of equation (1.1), where $p$ and $q: \mathbb{R}^{2} \rightarrow \mathbb{R}$ are continuous $\left(\omega_{1}, \omega_{2}\right)$-periodic functions. Theorems 1.2 and 1.4 , respectively, imply the following Corollaries 1.1 and 1.2. 
Corollary 1.1. Let inequalities (1.2) and (1.3) hold, and

$$
a \leq(-1)^{m+n} p(x, y) \leq b \text { for }(x, y) \in \mathbb{R}^{2},
$$

where $a$ and $b$ are positive constants. Then equation (1.16) has one and only one $\left(\omega_{1}, \omega_{2}\right)$-periodic solution.

Corollary 1.2. If

$$
(-1)^{m+n} p(x, y)<0 \quad \text { for } \quad(x, y) \in \mathbb{R}^{2}
$$

and inequalities (1.10) hold, then equation (1.16) has one and only one $\left(\omega_{1}, \omega_{2}\right)$ periodic solution.

\section{Auxiliary Statements}

2.1. Lemmas on a priori estimates. Denote by $C_{\omega_{1} \omega_{2}}^{k, l}$ the Banach space of continuous $\left(\omega_{1}, \omega_{2}\right)$-periodic functions $u$ having continuous partial derivatives $u^{(i, j)}(i=0, \ldots, k ; j=0, \ldots, l)$, with the norm

$$
\|u\|_{C_{\omega_{1} \omega_{2}}^{k, l}}=\max \left\{\sum_{i=0}^{k} \sum_{j=0}^{l}\left|u^{(i, k)}(x, y)\right|:(x, y) \in \Omega\right\} .
$$

Besides, we will use the notation $C_{\omega_{1} \omega_{2}}^{0,0}=C_{\omega_{1} \omega_{2}}$ and

$$
\|u\|_{L_{\omega_{1} \omega_{2}}^{2}}=\left(\int_{0}^{\omega_{1}} \int_{0}^{\omega_{2}} u^{2}(s, t) d s d t\right)^{\frac{1}{2}}
$$

Set

$$
\begin{gathered}
\mathcal{L}(u)(x, y)=u^{(2 m, 2 n)}(x, y)-\sum_{k=0}^{n-1}\left(a_{m k} u^{(2 m, 2 k)}(x, y)+b_{m k} u^{(2 m, 2 k+1)}(x, y)\right) \\
+\sum_{i=0}^{m-1}\left(a_{i n} u^{(2 i, 2 n)}(x, y)+c_{i n} u^{(2 i+1,2 n)}(x, y)\right) \\
+\sum_{i=0}^{m-1} \sum_{k=0}^{n-1}\left(a_{i k} u^{(2 i, 2 k)}(x, y)+b_{i k} u^{(2 i, 2 k+1)}(x, y)+c_{i k} u^{(2 i+1,2 k)}(x, y)\right),
\end{gathered}
$$

and consider the differential inequalities

$$
a|u(x, y)|-c \leq(-1)^{m+n} \mathcal{L}(u)(x, y) \operatorname{sgn} u(x, y) \leq b|u(x, y)|+c
$$

and

$$
(-1)^{m+n} \mathcal{L}(u)(x, y) u(x, y) \leq-g(x, y, u(x, y)),
$$

where $a>0, b \geq a, c \geq 0$ are constants and $g: \mathbb{R}^{3} \rightarrow \mathbb{R}$ is a continuous function such that

$$
g\left(x+\omega_{1}, y, z\right)=g(x, y, z), \quad g\left(x, y+\omega_{2}, z\right)=g(x, y, z) \quad \text { for } \quad(x, y, z) \in \mathbb{R}^{3}
$$

and

$$
g(x, y, z)>0 \text { for }(x, y) \in \mathbb{R}^{2},|z| \geq c .
$$


By a $\left(\omega_{1}, \omega_{2}\right)$-periodic solution of the differential inequality (2.2) (differential inequality (2.3)) we understand a function $u \in C_{\omega_{1} \omega_{2}}^{2 m, 2 n}$ satisfying this inequality everywhere in $\mathbb{R}^{2}$.

Lemma 2.1. If conditions (1.2) and (1.3) hold, then there exists a positive constant $r$ independent of $c$ such that an arbitrary $\left(\omega_{1}, \omega_{2}\right)$-periodic solution $u$ of the differential inequality (2.2) admits the estimate

$$
\|u\|_{C_{\omega_{1} \omega_{2}}} \leq r c \text {. }
$$

To prove the lemma we will need Lemmas 2.2-2.6 formulated below.

Lemma 2.2. Let $u \in C_{\omega_{1} \omega_{2}}^{1,1}$ and

$$
\left|u\left(x_{0}, y_{0}\right)\right|=\min \left\{|u(x, y)|:(x, y) \in \mathbb{R}^{2}\right\} .
$$

Then

and

$$
\left|u\left(x_{0}, y_{0}\right)\right| \leq\left(\omega_{1} \omega_{2}\right)^{-\frac{1}{2}}\|u\|_{L_{\omega_{1} \omega_{2}}^{2}}
$$

$$
\begin{aligned}
\|u\|_{C_{\omega_{1} \omega_{2}}} & \leq\left|u\left(x_{0}, y_{0}\right)\right|+\left(\frac{\omega_{2}}{\omega_{1}}\right)^{\frac{1}{2}}\left\|u^{(0,1)}\right\|_{L_{\omega_{1} \omega_{2}}^{2}} \\
& +\left(\frac{\omega_{1}}{\omega_{2}}\right)^{\frac{1}{2}}\left\|u^{(1,0)}\right\|_{L_{\omega_{1} \omega_{2}}^{2}}+2\left(\omega_{1} \omega_{2}\right)^{\frac{1}{2}}\left\|u^{(1,1)}\right\|_{L_{\omega_{1} \omega_{2}}^{2}} .
\end{aligned}
$$

The proof of the lemma is in [6].

Lemma 2.3. If $u: \mathbb{R} \rightarrow \mathbb{R}$ is a $k$-times continuously differentiable $\omega$-periodic function, then

$$
\int_{0}^{\omega}\left|u^{(i)}(s)\right|^{2} d s \leq\left(\frac{\omega}{2 \pi}\right)^{2 k-2 i} \int_{0}^{\omega}\left|u^{(k)}(s)\right|^{2} d s \quad(i=1, \ldots, k) .
$$

This is Wirtinger's lemma and one can find its proof in [5] (see also [7]).

Lemma 2.3 immediately implies

Lemma 2.4. If $u \in C_{\omega_{1} \omega_{2}}^{k, l}$, then

$$
\begin{aligned}
\left\|u^{(i, 0)}\right\|_{L_{\omega_{1} \omega_{2}}^{2}} & \leq\left(\frac{\omega_{1}}{2 \pi}\right)^{k-i}\left\|u^{(k, 0)}\right\|_{L_{\omega_{1} \omega_{2}}^{2}}, \quad\left\|u^{(0, j)}\right\|_{L_{\omega_{1} \omega_{2}}^{2}} \leq\left(\frac{\omega_{2}}{2 \pi}\right)^{l-j}\left\|u^{(0, l)}\right\|_{L_{\omega_{1} \omega_{2}}^{2}}, \\
\left\|u^{(i, j)}\right\|_{L_{\omega_{1} \omega_{2}}^{2}} & \leq\left(\frac{\omega_{1}}{2 \pi}\right)^{k-i}\left(\frac{\omega_{2}}{2 \pi}\right)^{l-j}\left\|u^{(k, l)}\right\|_{L_{\omega_{1} \omega_{2}}^{2}} \quad(i=1, \ldots, k ; j=1, \ldots, l) .
\end{aligned}
$$

Lemma 2.5. Let $\varepsilon$ be a positive constant and $u$ be a $\left(\omega_{1}, \omega_{2}\right)$-periodic solution of the differential inequality (2.2). Then the following inequalities hold in $\mathbb{R}^{2}$

$$
\begin{gathered}
u^{2}(x, y) \leq \frac{2}{a}|\mathcal{L}(u)(x, y) u(x, y)|+\frac{c^{2}}{a^{2}}, \\
(-1)^{m+n} \mathcal{L}(u)(x, y) u(x, y) \geq|\mathcal{L}(u)(x, y) u(x, y)|-\frac{c^{2}}{a}, \\
\mathcal{L}^{2}(u)(x, y)<(b+\varepsilon)|\mathcal{L}(u)(x, y) u(x, y)|+\gamma^{2} c^{2},
\end{gathered}
$$


where

$$
\gamma=(2 a \varepsilon)^{-\frac{1}{2}}(b+\varepsilon)
$$

Proof. From (2.2) we have

$$
(-1)^{m+n} \mathcal{L}(u)(x, y) u(x, y) \geq a u^{2}(x, y)-c|u(x, y)| \geq \frac{a}{2} u^{2}(x, y)-\frac{c^{2}}{2 a}
$$

which implies inequalities (2.7) and (2.8).

Let $(x, y) \in \mathbb{R}^{2}$ be an arbitrarily fixed point. If $u(x, y)=0$, then in view of (2.2) we have $|\mathcal{L}(u)(x, y)| \leq c$ and, consequently, inequality (2.9). Therefore it remains to consider the case, where $u(x, y) \neq 0$. Setting

$$
\begin{gathered}
\eta=\left((-1)^{m+n} \mathcal{L}(u)(x, y) \operatorname{sgn} u(x, y)-a|u(x, y)|+c\right)((b-a)|u(x, y)|+2 c)^{-1}, \\
p=a+(b-a) \eta, \quad q=(2 \eta-1) c \operatorname{sgn} u(x, y),
\end{gathered}
$$

and taking into account (2.2) we get

$$
a \leq p \leq b, \quad|q| \leq c .
$$

On the other hand, it is clear that

$$
(-1)^{m+n} \mathcal{L}(u)(x, y)=p u(x, y)+q .
$$

Therefore

$$
\begin{gathered}
\mathcal{L}^{2}(u)(x, y)=(-1)^{m+n} p \mathcal{L}(u)(x, y) u(x, y)+p q u(x, y)+q^{2} \\
\leq p|\mathcal{L}(u)(x, y) u(x, y)|+\frac{a}{2} \varepsilon u^{2}(x, y)+\frac{p^{2} q^{2}}{2 a \varepsilon}+q^{2} .
\end{gathered}
$$

Hence according to (2.7), (2.10) and (2.11) we get inequality (2.9).

The following lemma is an immediate consequence of the formula of integration by parts.

\section{Lemma 2.6. If}

$$
u \in C_{\omega_{1} \omega_{2}}^{2 m, 2 n}
$$

then for arbitrary $k \in\{0, \ldots, m\}, l \in\{0, \ldots, n\}, i \in\{0, \ldots, 2 m-2 k\}$ and $j \in\{0, \ldots, 2 n-2 l\}$

$$
\begin{gathered}
\int_{0}^{\omega_{1}} \int_{0}^{\omega_{2}} u^{(i, j)}(x, y) u^{(i+2 k, j+2 l)}(x, y) d x d y=(-1)^{k+l}\left\|u^{(i+k, j+l)}\right\|_{L_{\omega_{1} \omega_{2}}^{2}}^{2}, \\
\int_{0}^{\omega_{1}} \int_{0}^{\omega_{2}} u^{(i, j)}(x, y) u^{(i+2 k-1, j+2 l)}(x, y) d x d y=0, \\
\int_{0}^{\omega_{1}} \int_{0}^{\omega_{2}} u^{(i, j)}(x, y) u^{(i+2 k, j+2 l-1)}(x, y) d x d y=0 .
\end{gathered}
$$


Proof of Lemma 2.1. By (1.3) there exist numbers $\varepsilon>0$ and $\delta \in(0,1)$ such that

$$
\begin{gathered}
\sum_{k=0}^{n-1}\left(\frac{\omega_{2}}{2 \pi}\right)^{2 n-2 k}\left|a_{m k}\right|+\sum_{i=0}^{m-1}\left(\frac{\omega_{1}}{2 \pi}\right)^{2 m-2 i}\left|a_{i n}\right| \\
+\sum_{i=0}^{m-1} \sum_{k=0}^{n-1}\left(\frac{\omega_{1}}{2 \pi}\right)^{2 m-2 i}\left(\frac{\omega_{2}}{2 \pi}\right)^{2 n-2 k}\left|a_{i k}\right|+\left(\frac{\omega_{1}}{2 \pi}\right)^{m}\left(\frac{\omega_{2}}{2 \pi}\right)^{n}(b+\varepsilon)^{\frac{1}{2}}<1-\delta .
\end{gathered}
$$

Let $u$ be an arbitrary $\left(\omega_{1}, \omega_{2}\right)$-periodic solution of equation $(2.2)$. Then by Lemma 2.5, inequalities (2.7)-(2.9) hold, where $\gamma$ is the number given by (2.10).

By Lemma 2.6 and conditions (1.2), (2.1), we have

$$
\begin{gathered}
(-1)^{m+n} \int_{0}^{\omega_{1}} \int_{0}^{\omega_{2}} \mathcal{L}(u)(x, y) u(x, y) d x d y=\left\|u^{(m, n)}\right\|_{L_{\omega_{1} \omega_{2}}^{2}}^{2}-\sum_{i=0}^{m-1}\left|a_{i n}\right|\left\|u^{(i, n)}\right\|_{L_{\omega_{1} \omega_{2}}^{2}}^{2} \\
\quad-\sum_{k=0}^{n-1}\left|a_{m k}\right|\left\|u^{(m, k)}\right\|_{L_{\omega_{1} \omega_{2}}^{2}}^{2}-\sum_{i=0}^{m-1} \sum_{k=0}^{n-1}\left|a_{i k}\right|\left\|u^{(i, k)}\right\|_{L_{\omega_{1} \omega_{2}}^{2}}^{2} \\
\int_{0}^{\omega_{1}} \int_{0}^{\omega_{2}} \mathcal{L}(u)(x, y) u^{(2 m, 2 n)}(x, y) d x d y=\mu^{2}-\sum_{i=0}^{m-1}\left|a_{i n}\right|\left\|u^{(m+i, 2 n)}\right\|_{L_{\omega_{1} \omega_{2}}^{2}}^{2} \\
-\sum_{k=0}^{n-1}\left|a_{m k}\right|\left\|u^{(2 m, n+k)}\right\|_{L_{\omega_{1} \omega_{2}}^{2}}^{2}-\sum_{i=0}^{m-1} \sum_{k=0}^{n-1}\left|a_{i k}\right|\left\|u^{(m+i, n+k)}\right\|_{L_{\omega_{1} \omega_{2}}^{2}}^{2}
\end{gathered}
$$

where $\mu=\left\|u^{(2 m, 2 n)}\right\|_{L_{\omega_{1} \omega_{2}}^{2}}$. Hence by Lemma 2.4 and inequality (2.8) it follows that

$$
\begin{gathered}
\int_{0}^{\omega_{1}} \int_{0}^{\omega_{2}}|\mathcal{L}(u)(x, y) u(x, y)| d x d y+\left|a_{0 n}\right|\left\|u^{(0, n)}\right\|_{L_{\omega_{1} \omega_{2}}^{2}}^{2}+\left|a_{m 0}\right|\left\|u^{(m, 0)}\right\|_{L_{\omega_{1} \omega_{2}}^{2}}^{2} \\
\leq\left(\frac{\omega_{1}}{2 \pi}\right)^{2 m}\left(\frac{\omega_{2}}{2 \pi}\right)^{2 n} \mu^{2}+\frac{\omega_{1} \omega_{2}}{a} c^{2} \\
\mu^{2} \leq\left(\sum_{i=0}^{m-1} \sum_{k=0}^{n-1}\left(\frac{\omega_{1}}{2 \pi}\right)^{2 m-2 i}\left(\frac{\omega_{2}}{2 \pi}\right)^{2 n-2 k}\left|a_{i k}\right|+\sum_{k=0}^{n-1}\left(\frac{\omega_{2}}{2 \pi}\right)^{2 n-2 k}\left|a_{m k}\right|\right. \\
\left.+\sum_{i=0}^{m-1}\left(\frac{\omega_{1}}{2 \pi}\right)^{2 m-2 i}\left|a_{i n}\right|\right) \mu^{2}+\int_{0}^{\omega_{1}} \int_{0}^{\omega_{2}} \mathcal{L}(u)(x, y) u^{(2 m, 2 n)}(x, y) d x d y
\end{gathered}
$$

On the other hand, by Schwartz's inequality and inequality (2.9) we have

$$
\int_{0}^{\omega_{1}} \int_{0}^{\omega_{2}} \mathcal{L}(u)(x, y) u^{(2 m, 2 n)}(x, y) d x d y
$$




$$
\leq \mu(b+\varepsilon)^{\frac{1}{2}}\left(\int_{0}^{\omega_{1}} \int_{0}^{\omega_{2}}|\mathcal{L}(u)(x, y) u(x, y)| d x d y\right)^{\frac{1}{2}}+\gamma\left(\omega_{1} \omega_{2}\right)^{\frac{1}{2}} \mu c .
$$

If along with this we take into account inequalities (2.12) and (2.13), then from (2.14) we get

$$
\mu^{2}=(1-\delta) \mu^{2}+\delta r_{0} c, \quad \text { where } \quad r_{0}=\delta^{-1}\left((b+\varepsilon)^{\frac{1}{2}}\left(\frac{\omega_{1} \omega_{2}}{a}\right)^{\frac{1}{2}}+\gamma\left(\omega_{1} \omega_{2}\right)^{\frac{1}{2}}\right)
$$

and, consequently,

$$
\mu \leq r_{0} c
$$

Setting

$$
r_{1}=\left(\frac{\omega_{1}}{2 \pi}\right)^{m}\left(\frac{\omega_{2}}{2 \pi}\right)^{n} r_{0}+\left(\frac{\omega_{1} \omega_{2}}{a}\right)^{\frac{1}{2}}
$$

and applying Lemma 2.4 again, from (2.7), (2.13) and (2.15) we obtain

$$
\left\|u^{(i, j)}\right\|_{L_{\omega_{1} \omega_{2}}^{2}} \leq r_{i j} c \quad(i, j=0,1)
$$

where

$$
\begin{aligned}
& r_{00}=\left(\frac{2}{a}\right)^{\frac{1}{2}} r_{1}+a^{-1}\left(\omega_{1} \omega_{2}\right)^{\frac{1}{2}}, \quad r_{01}=\left|a_{0 n}\right|^{-\frac{1}{2}}\left(\frac{\omega_{2}}{2 \pi}\right)^{n-1} r_{1}, \\
& r_{10}=\left|a_{m 0}\right|^{-\frac{1}{2}}\left(\frac{\omega_{1}}{2 \pi}\right)^{m-1} r_{1}, \quad r_{11}=\left(\frac{\omega_{1}}{2 \pi}\right)^{m-1}\left(\frac{\omega_{2}}{2 \pi}\right)^{n-1} r_{0} .
\end{aligned}
$$

By Lemma 2.2, estimate (2.6) follows from (2.16), where

$$
r=\left(\omega_{1} \omega_{2}\right)^{-\frac{1}{2}} r_{00}+\left(\frac{\omega_{2}}{\omega_{1}}\right)^{\frac{1}{2}} r_{01}+\left(\frac{\omega_{1}}{\omega_{2}}\right)^{\frac{1}{2}} r_{10}+2\left(\omega_{1} \omega_{2}\right)^{\frac{1}{2}} r_{11}
$$

is a positive constant independent of $u$ and $c$.

Lemma 2.7. Let conditions (1.10), (2.4) and (2.5) hold. Then there exists a positive constant $r$ independent of $c$ such that an arbitrary $\left(\omega_{1}, \omega_{2}\right)$-periodic solution $u$ of the differential inequality (2.3) admits the estimate

$$
\|u\|_{C_{\omega_{1} \omega_{2}}} \leq r g^{*}(c)
$$

where

$$
g^{*}(c)=c+\max \left\{|g(x, y, z)|^{\frac{1}{2}}:(x, y) \in \mathbb{R}^{2},|z| \leq c\right\} .
$$

Proof. If we integrate inequality $(2.3)$ over $\left[0, \omega_{1}\right] \times\left[0, \omega_{2}\right]$, then in view of Lemma 2.6 and inequality $(2.11)$ we get

$$
\begin{aligned}
& \left\|u^{(m, n)}\right\|_{L_{\omega_{1} \omega_{2}}^{2}}^{2}+\sum_{i=0}^{m-1}\left|a_{i n}\right|\left\|u^{(i, n)}\right\|_{L_{\omega_{1} \omega_{2}}^{2}}^{2}+\sum_{k=0}^{n-1}\left|a_{m k}\right|\left\|u^{(m, k)}\right\|_{L_{\omega_{1} \omega_{2}}^{2}}^{2} \\
& +\sum_{i=0}^{m-1} \sum_{k=0}^{n-1}\left|a_{i k}\right|\left\|u^{(i, k)}\right\|_{L_{\omega_{1} \omega_{2}}^{2}}^{2}+\int_{0}^{\omega_{2} \omega_{1}} \int_{0}^{\omega_{1}} g(x, y, u(x, y)) d x d y \leq 0 .
\end{aligned}
$$

Hence by (2.5) and (2.18) it follows that

$$
\min \left\{|u(x, y)|:(x, y) \in \mathbb{R}^{2}\right\} \leq c,
$$




$$
\begin{gathered}
\left\|u^{(m, n)}\right\|_{L_{\omega_{1} \omega_{2}}^{2}} \leq\left(\omega_{1} \omega_{2}\right)^{\frac{1}{2}} g^{*}(c), \quad\left\|u^{(0, n)}\right\|_{L_{\omega_{1} \omega_{2}}^{2}} \leq\left|a_{0 n}\right|^{-1}\left(\omega_{1} \omega_{2}\right)^{\frac{1}{2}} g^{*}(c), \\
\left\|u^{(m, 0)}\right\|_{L_{\omega_{1} \omega_{2}}^{2}} \leq\left|a_{m 0}\right|^{-1}\left(\omega_{1} \omega_{2}\right)^{\frac{1}{2}} g^{*}(c) .
\end{gathered}
$$

Hence Lemma 2.4 yields

$$
\left\|u^{(i, j)}\right\|_{L_{\omega_{1} \omega_{2}}^{2}} \leq r_{i j} g^{*}(c) \quad(i, j=0,1)
$$

where

$$
\begin{gathered}
r_{10}=\left|a_{m 0}\right|\left(\omega_{1} \omega_{2}\right)^{\frac{1}{2}}\left(\frac{\omega_{1}}{2 \pi}\right)^{m-1}, \quad r_{01}=\left|a_{0 n}\right|\left(\omega_{1} \omega_{2}\right)^{\frac{1}{2}}\left(\frac{\omega_{2}}{2 \pi}\right)^{n-1}, \\
r_{11}=\left(\omega_{1} \omega_{2}\right)^{\frac{1}{2}}\left(\frac{\omega_{1}}{2 \pi}\right)^{m}\left(\frac{\omega_{2}}{2 \pi}\right)^{n} .
\end{gathered}
$$

By Lemma 2.2, (2.19) and (2.20) imply estimate (2.6), where

$$
r=1+\left(\frac{\omega_{2}}{\omega_{1}}\right)^{\frac{1}{2}} r_{01}+\left(\frac{\omega_{1}}{\omega_{2}}\right)^{\frac{1}{2}} r_{10}+2\left(\omega_{1} \omega_{2}\right)^{\frac{1}{2}} r_{11}
$$

is a constant independent of $u$ and $c$.

2.2. Lemmas on the solvability of linear and nonlinear periodic problems. Consider the linear nonhomogeneous and homogeneous hyperbolic equations

$$
\begin{gathered}
\mathcal{L}(u)=(-1)^{m+n} b u+q(x, y), \\
\mathcal{L}(u)=(-1)^{m+n} b u
\end{gathered}
$$

and the linear homogeneous ordinary differential equations

$$
\begin{gathered}
v^{(2 m)}=\sum_{i=0}^{m-1}\left(a_{i n} v^{(2 i)}+c_{i n} v^{(2 i+1)}\right), \\
w^{(2 n)}=\sum_{k=0}^{n-1}\left(a_{m k} w^{(2 k)}+b_{m k} W^{(2 k+1)}\right),
\end{gathered}
$$

where $\mathcal{L}$ is the differential operator given by equality (2.1) and $b$ is some constant different from zero.

Lemma 2.8. Let either $b>0$ and inequalities (1.2) and (1.3) or $b<0$ and inequalities (1.10) hold. Then there exists a linear bounded operator $\mathcal{G}: C_{\omega_{1} \omega_{2}} \rightarrow$ $C_{\omega_{1} \omega_{2}}^{2 m, 2 n}$ such that for any $q \in C_{\omega_{1} \omega_{2}}$ equation $(2.21)$ has a unique $\left(\omega_{1}, \omega_{2}\right)$-periodic solution

$$
u(x, y)=\mathcal{G}(q)(x, y) \quad \text { for } \quad(x, y) \in \mathbb{R}^{2} .
$$

Proof. By Theorem 1.1 from [6], to prove the lemma it is sufficient to show that equation $(2.22)$ has only a trivial $\left(\omega_{1}, \omega_{2}\right)$-periodic solution, and equation (2.23) (equation $(2.24))$ has only a trivial $\omega_{1}$-periodic $\left(\omega_{2}\right.$-periodic) solution.

Let $u, v$ and $w$ be, respectively, a $\left(\omega_{1}, \omega_{2}\right)$-periodic, a $\omega_{1}$-periodic and a $\omega_{2}$ periodic solution of equations $(2.22),(2.23)$ and (2.24). Our goal is to prove that $u(x, y) \equiv 0, v(t) \equiv 0, w(t) \equiv 0$. 
First consider the case where $b>0$ and conditions (1.2) and (1.3) hold. Then $u$ is a solution of the differential inequality (2.2), where $a=b$ and $c=0$. Hence by Lemma 2.1, we get that $u(x, y) \equiv 0$. Multiplying (2.23) and (2.24), respectively, by $v^{(2 m)}(t)$ and $w^{(2 n)}(t)$ and integrating over $\left[0, \omega_{1}\right]$ and $\left[0, \omega_{2}\right]$, we get

$$
\begin{aligned}
& \int_{0}^{\omega_{1}}\left|v^{(2 m)}(t)\right|^{2} d t=\sum_{i=0}^{m-1}(-1)^{m+i} a_{i n} \int_{0}^{\omega_{1}}\left|v^{(m+i)}(t)\right|^{2} d t, \\
& \int_{0}^{\omega_{2}}\left|w^{(2 n)}(t)\right|^{2} d t=\sum_{i=0}^{n-1}(-1)^{n+k} a_{m k} \int_{0}^{\omega_{2}}\left|w^{(n+k)}(t)\right|^{2} d t .
\end{aligned}
$$

Hence by Lemma 2.3 and inequality (1.3) it follows that

$$
\int_{0}^{\omega_{1}}\left|v^{(2 m)}(t)\right|^{2} d t \leq \alpha \int_{0}^{\omega_{1}}\left|v^{(2 m)}(t)\right|^{2} d t, \quad \int_{0}^{\omega_{2}}\left|w^{(2 n)}(t)\right|^{2} d t \leq \beta \int_{0}^{\omega_{2}}\left|w^{(2 n)}(t)\right|^{2} d t,
$$

where

$$
\alpha=\sum_{i=0}^{m-1}\left(\frac{\omega_{1}}{2 \pi}\right)^{2 m-2 i}\left|a_{i n}\right|<1, \quad \beta=\sum_{i=0}^{n-1}\left(\frac{\omega_{2}}{2 \pi}\right)^{2 n-2 k}\left|a_{m k}\right|<1 .
$$

Therefore it is clear that $v^{(2 m)}(t) \equiv 0, w^{(2 n)}(t) \equiv 0$ and, consequently, $v(t) \equiv$ const and $w(t) \equiv$ const. Taking into account the fact that $a_{0 n} \neq 0$ and $a_{m 0} \neq 0$, from (2.23) and (2.24) we conclude that $v(t) \equiv 0$ and $w(t) \equiv 0$.

Now consider the case, where $b<0$ and inequalities (1.10) hold. Then $u$ is a solution of the differential inequality (2.3), where $g(x, y, z) \equiv|b| z^{2}$. Hence by Lemma 2.7, it follows that $u(x, y) \equiv 0$. On the other hand, using inequalities (1.10), from (2.23) and (2.26) $((2.4)$ and (2.27)) we obtain that $v(t) \equiv 0(w(t) \equiv$ $0)$.

Lemma 2.9. Let either $b>0$ and inequalities (1.2) and (1.3) or $b<0$ and inequalities (1.10) hold. Moreover, let there exist a positive constant $\rho$ such that for any $\lambda \in(0,1)$ every $\left(\omega_{1}, \omega_{2}\right)$-periodic solution of the differential equation

$$
\mathcal{L}(u)=(-1)^{m+n}(1-\lambda) b u+\lambda f(x, y, u)
$$

admits the estimate

$$
\|u\|_{C_{\omega_{1} \omega_{2}}^{2 m-1,2 n-1}} \leq \rho .
$$

Then equation (1.1) has at least one $\left(\omega_{1}, \omega_{2}\right)$-periodic solution.

This lemma immediately follows from Lemma 2.8 and Theorem 2.1 from [6].

\section{Proofs of the Main Results}

Proof of Theorem 1.1. Let $r$ and $\mathcal{G}$ be the number and the operator appearing in Lemmas 2.1 and 2.8, and let $\|\mathcal{G}\|$ be the norm of the operator $\mathcal{G}$. Set

$$
\rho_{0}=\max \left\{b r c+|f(x, y, z)|:(x, y) \in \mathbb{R}^{2},|z| \leq r c\right\}, \quad \rho=\|\mathcal{G}\| \rho_{0} .
$$


By Lemma 2.9 and conditions (1.2), (1.3), to prove the theorem it is sufficient to show that for any $\lambda \in(0,1)$ an arbitrary $\left(\omega_{1}, \omega_{2}\right)$-periodic solution $u$ of equation (2.28) admits estimate (2.29).

According to (1.4) $u$ is a solution of the differential inequality (2.2). Hence by Lemma 2.1 we get estimate (2.6). On the other hand, by Lemma 2.8 we have the representation

$$
u(x, y)=\mathcal{G}\left(\lambda f(\cdot, \cdot, u)-(-1)^{m+n} \lambda u\right)(x, y) .
$$

By (2.6) and (3.1), the latter representation immediately implies estimate (2.29).

Proof of Theorem 1.2. Inequality (1.4) follows from (1.5), where

$$
c=\max \left\{|f(x, y, 0)|:(x, y) \in \mathbb{R}^{2}\right\} .
$$

However, by Theorem 1.1, this inequality along with conditions (1.2) and (1.3) guarantees the existence of $\left(\omega_{1}, \omega_{2}\right)$-periodic solution of equation (1.1). It remains to prove that $(1.1)$ has at most one $\left(\omega_{1}, \omega_{2}\right)$-periodic solution. Let $u_{1}$ and $u_{2}$ be arbitrary $\left(\omega_{1}, \omega_{2}\right)$-periodic solutions of that equation and

$$
u(x, y)=u_{1}(x, y)-u_{2}(x, y) .
$$

Then in view of $(1.5)$ the function $u$ is a $\left(\omega_{1}, \omega_{2}\right)$-periodic solution of the differential inequality (2.2), where $c=0$. Hence according to (1.2), (1.3) and Lemma 2.1 it follows that $u(x, y) \equiv 0$, i.e., $u_{1}(x, y) \equiv u_{2}(x, y)$.

We omit the proof of Theorem 1.3, since it can be proved in much the same way as Theorem 1.1. The only difference is that the constant $b$ should be an arbitrary negative number, and instead of Lemma 2.1 one should use Lemma 2.7, where

$$
g(x, y, z)=\min \left\{|b| z^{2},(-1)^{m+n+1} f(x, y, z)\right\} .
$$

Theorem 1.4 immediately follows from Theorem 1.3 and Lemma 2.7.

\section{REFERENCES}

1. A. K. AzIZ and M. G. Horak, Periodic solutions of hyperbolic partial differential equations in the large. SIAM J. Math. Anal. 3(1972), No. 1, 176-182.

2. L. CESARI, Existence in the large of periodic solutions of hyperbolic partial differential equations. Arch. Rational Mech. Anal. 20(1965), No. 2, 170-190.

3. L. CESARI, Smoothness properties of periodic solutions in the large of nonlinear hyperbolic differential systems. Funkcial. Ekvac. 9(1966), 325-338.

4. J. K. Hale, Periodic solutions of a class of hyperbolic equations containing a small parameter. Arch. Rational Mech. Anal. 23(1966), 380-398.

5. G. H. Hardy, J. E. Littlewood, and G. Pólya, Inequalities. Cambridge Univ. Press, Cambridge, 1934.

6. I. Kiguradze and T. Kiguradze, On solvability of boundary value problems for higher order nonlinear hyperbolic equations. Nonlinear Anal. (submitted).

7. I. T. Kiguradze and T. KusAno, On periodic solutions of higher-order nonautonomous ordinary differential equations. (Russian) Differ. Uravn. 35(1999), No. 1, 72-78; English transl.: Differential Equations 35(1999), No. 1, 71-77 
8. T. Kiguradze, On periodic in the plane solutions of second order linear hyperbolic systems. Arch. Math. (Brno) 33(1997), No. 4, 253-272.

9. T. Kiguradze, On unique solvability of the periodic problem in the plane for linear hyperbolic equations. Mem. Differential Equations Math. Phys. 10(1997), 129-131.

10. T. Kiguradze, On doubly periodic solutions of a class of nonlinear hyperbolic equations. (Russian) Differ. Uravn. 34(1998), No. 2, 238-245; English transl.: Differential Equations 34(1998), No. 2, 242-249.

11. T. Kiguradze, On periodic in the plane solutions of nonlinear hyperbolic equations. Nonlinear Anal. 39(2000), No. 2, Ser. A: Theory Methods, 173-185.

12. T. Kiguradze and V. Lakshmikantham, On doubly periodic solutions of fourth-order linear hyperbolic equations. Nonlinear Anal. 49(2002), No. 1, Ser. A: Theory Methods, $87-112$.

13. T. Kiguradze and T. Smith, On doubly periodic solutions of quasilinear hyperbolic equations of the fourth order. Proc. Confer. Differential \& Difference Equations Appl., 541-553. Melbourne, Florida, 2006.

14. J. Mawhin, Solutions périodiques d'équations aux dérivées partielles hyperboliques non linéaires. Miscellanea, 301-315, Presses Univ. Bruxelles, Brussels, 1978.

(Received 11.06.2007)

Author's address:

Florida Institute of Technology

Department of Mathematical Sciences

$150 \mathrm{~W}$ University Blvd.

Melbourne, Fl 32901

USA

E-mail: tkigurad@fit.edu 